\title{
Calcium influx kinetics, and the features of potassium channels of peripheral lymphocytes in primary Sjögren's syndrome
}

\author{
Nóra Legány ${ }^{\mathrm{a}}$, Gergely Toldi ${ }^{\mathrm{b}}$, Csaba Orbán ${ }^{\mathrm{c}}$, Nóra Megyes ${ }^{\mathrm{a}}$, Anna Bajnok ${ }^{\mathrm{b}}$, Attila Balog a,* \\ a Department of Rheumatology, Faculty of Medicine, Albert Szent-Györgyi Health Center, University of Szeged Kálvária sgt. 57., H-6725, Szeged, Hungary \\ b First Department of Obstetrics and Gynecology, Faculty of Medicine, Semmelweis University, Budapest, Baross u. 27., H-1088, Budapest, Hungary

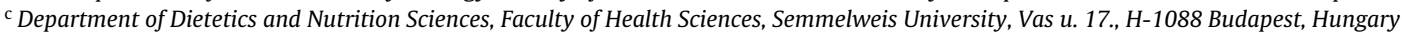

\section{A R T I C L E I N F O}

\section{Article history:}

Received 17 March 2016

Received in revised form 1 June 2016

Accepted 9 June 2016

Available online $\mathrm{xxx}$

\section{Keywords:}

Primary Sjögren's syndrome

Calcium influx

Potassium channels

Lymphocyte activation

\begin{abstract}
A B S T R A C T
Objective: The transient increase of the cytoplasmic free calcium level plays a key role in the process of lymphocyte activation. Kv1.3 and IKCa1 potassium channels are important regulators of the maintenance of calcium influx and present a possible target for selective immunomodulation.

Design: Case-control study.

Subjects and methods: We took peripheral blood samples from 8 healthy individuals and 15 primary Sjögren's syndrome (pSS) patients. We evaluated calcium influx kinetics following activation in peripheral T lymphocytes. We also assessed the sensitivity of T lymphocytes to specific inhibition of the Kv1.3 and IKCa1 potassium channels, and the Kv1.3 channel expression.

Results: The basal cytoplasmatic calcium levels were lower in both Th1 and Th2 lymphocytes in pSS compared to controls. The peak of calcium influx in lymphocytes isolated from pSS patients is reached later, indicating that they respond more slowly to stimulation compared to controls. In healthy individuals, the inhibition of the IKCa1 channel decreased calcium influx in Th2 and CD4 cells to a lower extent than in Th1 and CD8 cells. On the contrary, the inhibition of Kv1.3 channels resulted in a larger decrease of calcium entry in Th2 and CD4 than in Th1 and CD8 cells. In the pSS group, neither of the inhibitors induced alteration in calcium influx. Expression of Kv1.3 channels on CD4, Th2 and CD8 lymphocytes in pSS was significantly higher compared to controls.

Conclusion: The altered expression and specific inhibition of potassium channels seem to be related to altered calcium influx kinetics in pSS which distinguish pSS either from healthy controls or other systemic autoimmune diseases.
\end{abstract}

(c) 2016 Elsevier GmbH. All rights reserved.

\section{Introduction}

Pathways of calcium homeostasis participate in a number of cellular processes that determine short and long-term function of $\mathrm{T}$ lymphocytes. The increase of the cytoplasmic calcium concentration from intra- and extracellular sources (i.e., the endoplasmic reticulum and store-operated calcium entry through the plasma

Abbreviations: AUC, area under the curve; $\left[\mathrm{Ca}^{2+}\right]_{c y t}$, cytoplasmic free calcium level; CRAC, calcium release activated calcium; Max, maximum value; MGTX, margatoxin; PBMC, peripheral blood mononuclear cell; PHA, phytohemagglutinin; pSS, primary Sjögren's syndrome; RA, rheumatoid arthritis; TCM, central memory T cell; TEM, effector memory $\mathrm{T}$ cell; $\mathrm{Th}$, $\mathrm{T}$ helper; $\mathrm{t}_{\max }$, time to reach maximum value; TRAM, triarylmethane.

* Corresponding author.

E-mail address: balog.attila@med.u-szeged.hu (A. Balog). membrane) is the cornerstone of T lymphocyte activation and functionality. Over the recent year, an increasing number of calcium channels and transporters have been described that play a key role in balancing cytoplasmic calcium levels in T cells. (Toldi, 2013; Feske, 2013).

In the course of lymphocyte activation, potassium channels maintain the driving force for sustained calcium influx from the extracellular milieu as they grant the efflux of potassium from the cytoplasm, thus conserving an electrochemical potential gradient between the intra- and extracellular spaces. There are two major types of potassium channels in T cells: the voltage gated Kv1.3 and the calcium-activated IKCa1 channels (Orbán et al., 2013). The relation between the calcium currents through calcium release activated calcium (CRAC) channels and the efflux of potassium makes the proliferation and activation of lymphocytes sensitive to pharmacological modulation of Kv1.3 and IKCa1 channels, and 
provides an opportunity for targeted intervention. Specific inhibition of these channels results in a diminished calcium influx in lymphocytes and a lower level of lymphocyte activation. Previous data suggest that selective modulation of lymphocyte activation through specific inhibition of potassium channels may be a possible therapeutic approach for the treatment of autoimmune disease (Beeton et al., 2006; Rangaraju et al., 2009).

Furthermore, a different characteristic potassium channel phenotype of effector memory $\mathrm{T}$ cells was described in multiple sclerosis (MS): terminally differentiated effector memory T (TEM) cells exhibit Kv1.3high IKCa1low channel phenotype, contrasting naïve, and central memory $\mathrm{T}$ cells, which exhibit a Kv1.3low IKCa1high channel phenotype (Wulff et al., 2003).

Although results from animal models are promising, limited data is available on the effects of potassium channel inhibition on $\mathrm{T}$ cell function in humans. Furthermore, besides naïve and memory $\mathrm{T}$ cells, alterations in the activation pattern of effector (CD4+ helper and CD8+ cytotoxic) T lymphocytes have not been described upon Kv1.3 and IKCa1 inhibition. Although these cells might have a lessspecific role in the maintenance of autoreactivity compared to TEM cells, their inhibition have important consequences on the overall immune response.

Therefore, over the recent years, we have investigated calcium influx characteristics in effector $\mathrm{T}$ cell subsets in a number of autoimmune diseases (Toldi et al., 2010, 2011, 2013, 2015).

In this study we focused on primary Sjögren's syndrome (pSS). pSS is a chronic autoimmune inflammatory disorder that primarily affects exocrine glands leading to their functional impairment. Although pSS etiology is not fully elucidated, it is well established that the interplay between genetic, environmental and hormonal factors represents the trigger of aberrant autoimmune response with $\mathrm{B}$ and $\mathrm{T}$ lymphocyte hyperactivity, autoantibody production and progressive destruction of target organs. The pathological hallmark of pSS is a chronic mononuclear cell infiltrate affecting exocrine glands mainly driven by $\mathrm{T}$ helper (Th) 1-type cytokines. The innate and adaptive immune responses both play an important role in this pathological process. Growing evidence suggests that CD4+ Th cells are predominant, and the Th1/Th2 balance shifts in favor of Th1 in gland, but Th2 cytokine repertoire prevails in sera (Tzioufas et al., 2012).

Therefore, in this study we aimed to characterize the effects of lymphocyte potassium channel inhibition on short-term peripheral blood T lymphocyte activation in major lymphocyte subsets of patients diagnosed with pSS. We employed a kinetic flow cytometry method to describe calcium influx characteristics of the CD4, Th1, Th2 and CD8 subsets and its sensitivity to the inhibition of Kv1.3 and IKCa1 lymphocyte potassium channels.

\section{Materials and methods}

\subsection{Patients}

We enrolled 8 healthy individuals and 15 patients with primary Sjögren's Syndrome (pSS). pSS patients fulfilling American European Consensus group (AECG) 2002 or American College of Rheumatology (ACR) 2012 classification criteria were included (Vitali et al., 2002; Shiboski et al., 2012). Clinical parameters of study participants are summarized in Table 1 . The following clinical symptoms occurred in pSS patients history: sicca parameters (100\%), cytopenia and anemia of autoimmune origin (87\%), arthritis (67\%), vasculitis (53\%), anti-SSA/B antibody positivity (100\%), low C3/C4 complement level (47\%), renal involvement (13\%), fever and night sweats (20\%), lymphadenopathy (20\%), lymphoma (0\%), weight loss (33\%), myositis (7\%), peripheral neuropathy (20\%). The EULAR Sjögren's syndrome disease activity (ESSDAI) scores were
Table 1

Clinical characteristics of study participants.

\begin{tabular}{lll}
\hline Characteristics & Healthy individuals $\mathrm{n}=8$ & pSS patients $\mathrm{n}=15$ \\
\hline Age (years) & $53(42-61)$ & $52(36-77)$ \\
Gender (male/female) & $2 / 6$ & $1 / 14$ \\
pSS duration (years) & - & $5(2-14)$ \\
Anti-SSA/B positivity & - & 15 \\
LSG biopsy positivity & - & 12 \\
ESSDAI & $8(5-12)$ & $2(0-4)$ \\
ESR $(\mathrm{mm} / \mathrm{h})$ & $27^{*}(7-55)$ \\
\hline
\end{tabular}

Data are expressed as median (interquartile range) for continous variables and as number for categorical variables. pSS, primary Sjögren's Syndrome; LSG biopsy, focal lymphocytic sialadenitis (FLS) with focus score $\geq 1$ in labial salivary gland (LSG) biopsy; Anti-SSA/B anti-SSA/B autoantibodies; ESSDAI, EULAR Sjögren's syndrome disease activity; ESR, erythrocyte sedimentation rate.

$\mathrm{p}<0.05$ vs. healthy individuals.

calculated at the time of sampling (Seror et al., 2015). The ESSDAI score was 2 corresponding to low disease activity. All pSS patients received a variety of disease modifying antirheumatic drugs (DMARDs), containing at least one of the following components: chloroquine, methylprednisolone, methotrexate and azathioprine.

Healthy controls had a negative history of rheumatic symptoms and negative status upon detailed physical and laboratory examination. No co-morbidities were detected in patients and controls that could have influenced our investigation, nor did they take any medication that could have interfered with the measurements. Written informed consent was obtained from all subjects, and our study was reviewed and approved by an independent ethical committee of the university. Laboratory studies and interpretations were performed on coded samples lacking personal and diagnostic identifiers. The study was adhered to the tenets of the most recent revision of the Declaration of Helsinki.

\subsection{Fluorescent staining}

Our measurements were carried out as described earlier (Toldi et al., 2011). Briefly, peripheral blood mononuclear cells (PBMCs) were isolated by a standard density gradient centrifugation from $9 \mathrm{~mL}$ of freshly drawn peripheral venous blood and afterwards kept in a modified RPMI medium (calcium concentration: $2 \mathrm{mM}$ ) throughout the following steps of the procedure. PBMCs were then incubated with the following conjugated anti-human monoclonal antibodies in order to differentiate T lymphocyte subsets: anti-CD4 PE-Cy7, anti-CD8 APC-Cy7, anti-CXCR3 APC (for the determination of Th1 cells) and anti-CCR4 PE (for the deter-mination of Th2 cells) (all from PharMingen, San Diego, CA, USA), as well as anti-Kv1.3 channel FITC (Sigma-Aldrich, St. Louis, MO, USA), according to the manufacturers' instructions. For monitoring [Ca2+]cyt, PBMCs were loaded with calcium sensitive Fluo-3 and Fura-Red dyes according to the manufacturer's recommendations (Invitrogen, Carlsbad, CA, USA).

\subsection{Flow cytometry}

PBMCs were equally distributed into three vials. The first vial was used as control. The second vial was treated with margatoxin (MGTX, $60 \mathrm{nM}$ ), a selective blocker of the Kv1.3 channel. The third vial was treated with a triarylmethane compound (TRAM, $60 \mathrm{nM}$ ), a specific inhibitor of the IKCa1 channel. PBMCs were activated by the addition of $20 \mu \mathrm{g}$ phytohemagglutinin (PHA) and the measurements were initiated directly afterwards on a BD FACSAria flow cytometer. Cell fluorescence data were measured and recorded for $15 \mathrm{~min}$ in a kinetic manner. 
Table 2

Prevalence of lymphocyte subsets in the overall lymphocyte population gated according to Forward Scatter Characteristics (FSC) and Side Scatter Characteristics (SSC) in 8 healthy individuals and 15 pSS patients.

\begin{tabular}{lll}
\hline Subset & Healthy & pSS \\
\hline CD4+/ly & $37.7(32.4-44.6)$ & $43.4(30.6-52.1)$ \\
CXCR3+/CD4 & $29.8(17.6-34.7)$ & $20.4(15.7-22.9)$ \\
CCR4+/CD4 & $18.9(12.5-26.3)$ & $13.1(8.8-23.6)$ \\
CD4+ CXCR3+/CD4+ CCR4+ ratio & $1.89(1.27-2.88)$ & $1.25(0.84-2.56)$ \\
CD8+/ly & $15.4(9.02-25.6)$ & $17.7(14.6-23.3)$ \\
\hline
\end{tabular}

Data are expressed as median [interquartile range]. CD4+ CXCR3+-Th1 subset, CD4+ CCR4+-Th2 subset, ly-overall lymphocyte population, pSS-primary Sjögren's syndrome.

\subsection{Data evaluation}

Recordings were evaluated with our specific software (FacsKin), based on the calculation of a double-logistic function for each recording (Toldi et al., 2011). This function is used to characterize measurements that have an increasing and a decreasing intensity as time passes. The software also calculated parameter values describing each function, such as the Area Under the Curve (AUC), Maximum (Max), time to reach maximum (tmax), and Slope values. AUC values correspond to the sum of [Ca2+]cyt increase, which further corresponds to the level of lymphocyte activation. Max values represent the peak value of the calcium influx curve upon lymphocyte activation. Tmax values describe how soon the peak value of the calcium influx curve is reached. The Slope value reflects how rapidly the peak of calcium influx is reached. A detailed description of the evaluation process can be found at www.facskin.com.

Statistics Data are expressed as median and interquartile range. Comparisons between two sample groups were made with the Mann-Whitney test. For comparisons between paired values in the same group Wilcoxon tests were applied. P values less than 0.05 were considered significant. Statistics were calculated using the R software.

\section{Results}

\subsection{Clinical data}

As seen in Table 1, the age and gender distribution of participants were similar in both study groups. Inflammatory parameters (erythrocyte sedimentation rate and $C$ reactive protein values) were higher in pSS patients than in healthy controls.

The number of patients in different subgroups according to therapeutic intervention was limited, and no significant differences in the investigated parameters were observed between the subgroups.

\subsection{The frequency of the investigated lymphocyte subsets}

We determined the frequency of CD4, Th1, Th2 and CD8, cells in samples of both study groups. CD4+ CXCR3+ cells were regarded as Th1 lymphocytes, while CD4+ CCR4+ cells were regarded as the Th2 subset. It was no detected significant difference regarding individual cell subsets among the study groups (Table 2 ).

\subsection{Basal cytoplasmatic calcium levels in the investigated lymphocyte subsets}

We evaluated the ratio of the basal median fluorescence of calcium binding dyes in lymphocytes of both study groups. Before the lymphocyte activation, the basal median fluorescence of calcium binding dyes was lower in both Th1 and Th2 lymphocyte subsets in the pSS group (CD4+ CXCR3: 24,494 [12,365-50,658], CD4+ CCR4+:

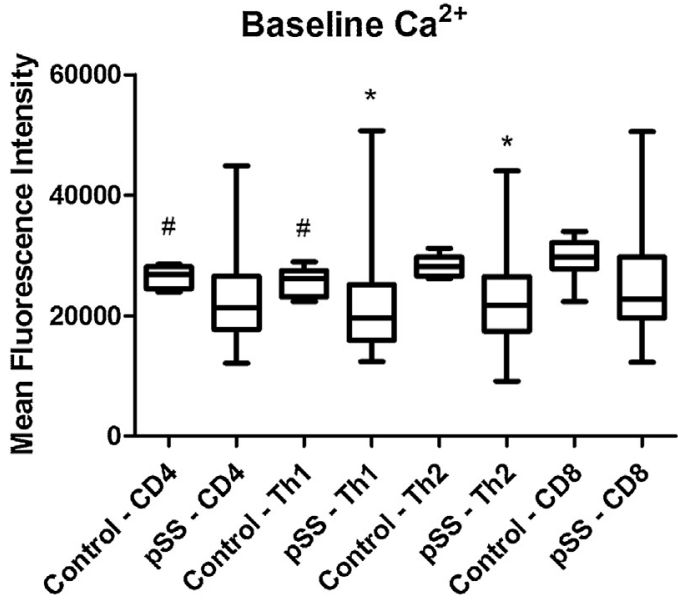

Fig. 1. The basal mean fluorescence intensity of calcium binding dyes in the investigated lymphocyte subsets in healthy individuals and primary Sjögren's syndrome patients. ${ }^{*} \mathrm{p}<0.05$ vs. control \# $\mathrm{p}<0.05$ vs CD8.

22768 [9110-44,026]) than in the healthy group CD4+ CXCR3: 26,151 [22,324-28,949], CD4 + CCR4: 28,187 [26,149-31,163] arbitrary units, $\mathrm{p}<0.05$ (median [interquartile range]) respectively (Fig. 1).

There was no difference between the study groups in CD8+ pSS: 26,483 [12,251-50,510], healthy: 29,797 [22,307-33,994] lymphocyte fluorescence. The basal cytoplasmatic calcium level was higher in the CD8+ subsets than in the CD4+ and Th1 subsets in the control group (CD8+: 29,797 [22,307-33,994], CD4+: 26,843 [23,925-28,502], CD4+CXCR3: 26,151 [22,324-28,949] $\mathrm{p}<0.05$ (median [interquartile range])), but in the pSS samples, there was no difference among the lymphocyte subsets (CD4+: 25,167 [12,126-44,988], CD4+ CXCR3: 24,494 [12,365-50,658], CD4+ CCR4: 22,768 [9110-44,026], CD8+: 26,483 [12,251-50,510] (median [interquartile range])) (Fig. 1).

\subsection{Calcium influx kinetics}

After lymphocyte activation with PHA, intracellular calcium influx kinetics were measured with calculated parameter values (AUC, Max, tmax, and slope) in healthy subjects and in pSS patients (Table 3). In the CD4+ population, AUC and Max values were lower in the pSS group compared to the healthy group. For Th1 lymphocytes, the AUC value was lower in pSS samples than in the control group. In the case of Th2 and CD8+ lymphocytes, the calculated parameter values showed no significant difference among the study groups (Table 3, Fig. 2).

\subsection{The effects of potassium channel inhibitors on lymphocyte calcium influx}

We measured the effect of potassium channel inhibitors (MGTX, TRAM) in both pSS and healthy groups. Our results revealed that the sensitivity of Th1 and Th2 cells to IKCa1 channel inhibition was different in lymphocytes isolated from healthy individuals (Table 3 ). Treatment with TRAM, the specific inhibitor of the IKCa1 channel decreased calcium influx in Th2 cells to a lower extent than in Th1 cells. On the contrary to IKCa1, the inhibition of Kv1.3 channels resulted in a larger decrease of calcium entry in Th2 than in Th1 cells. In healthy individuals, CD4 cells were more sensitive to the inhibition of Kv1.3 channels than the CD8 subset, responding with a higher level of decrease of the AUC value upon the application of MGTX. However, upon treatment with TRAM, CD8 cells showed a larger decrease in AUC and Max values than CD4 cells (Table 3). 
Table 3

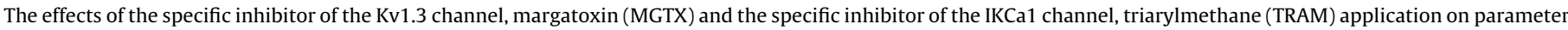

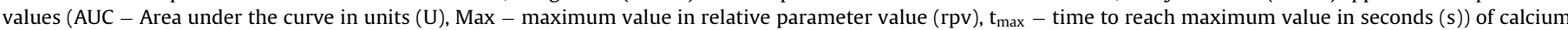
influx kinetics in peripheral lymphocytes obtained from 8 healthy individuals and 15 pSS patients.

\begin{tabular}{|c|c|c|c|c|c|c|c|}
\hline \multirow[b]{2}{*}{ Subset } & & \multicolumn{2}{|c|}{ No inhibitor } & \multicolumn{2}{|c|}{ MGTX (60 nM) } & \multicolumn{2}{|c|}{ TRAM (60 nM) } \\
\hline & & Healthy & pSS & Healthy & pSS & Healthy & pSS \\
\hline \multirow[t]{3}{*}{ CD4+ } & AUC (U) & 2591 (2349-2939) & $2033^{a}$ (1936-2255) & $2328^{\mathrm{b}}(2226-2353)$ & $2110(2043-2168)$ & $2483(2325-2723)$ & 2084 (1923-2319) \\
\hline & $\operatorname{Max}(\mathrm{rpv})$ & $1.46(1.249-1.608)$ & $1.115^{\mathrm{a}}(1.036-1.215)$ & $1.207^{\mathrm{b}}(1.141-1.22)$ & $1.085(1.049-1.121)$ & $1.303(1.198-1.483)$ & $1.143(1.033-1.227)$ \\
\hline & $\mathrm{t}_{\max }(\mathrm{s})$ & $631.9(471.7-930.1)$ & $504.3(355.4-576.3)$ & $913.5(666.2-1351)$ & $598.4(427.2-799.3)$ & $755.2(564.4-972.7)$ & $464.2(131.6-578.4)$ \\
\hline \multirow[t]{3}{*}{$\mathrm{CD} 4+\mathrm{CXCR} 3+$} & AUC (U) & $2411(2325-2508)$ & $2162^{\mathrm{a}}(1896-2358)$ & $2358(2336-2511)$ & 2067 (1972-2142) & $2204^{\mathrm{b}}(2151-2235)$ & $2104(1958-2262)$ \\
\hline & $\operatorname{Max}(\mathrm{rpv})$ & $1.284(1.203-1.335)$ & $1.122(1.022-1.298)$ & $1.134^{\mathrm{b}}(1.096-1.155)$ & $1.081(1.053-1.166)$ & $1.231(1.199-1.34)$ & $1.066(1.024-1.198)$ \\
\hline & $\mathrm{t}_{\max }(\mathrm{s})$ & $674.8(472.7-1003)$ & $552.8(189.1-646.6)$ & $901.6(756-990)$ & $604.1(432.5-782.3)$ & $872.9(645.3-1096)$ & $652.4(511.2-1100)$ \\
\hline \multirow[t]{3}{*}{ CD4+ CCR4+ } & AUC (U) & $2289(2158-2521)$ & $1984(1718-2396)$ & $2136^{\mathrm{b}}(2086-2189)$ & $2204(1737-2331)$ & $2306(2136-2396)$ & 2297 (2027-2537) \\
\hline & $\operatorname{Max}(\mathrm{rpv})$ & $1.239(1.099-1.361)$ & $1.097(1.006-1.222)$ & $1.088(1.057-1.12)$ & $1.278(1.144-1.359)$ & $1.192(1.09-1.249)$ & $1.164(1.104-1.407)$ \\
\hline & $\mathrm{t}_{\max }(\mathrm{s})$ & $791.1(626.6-1046)$ & $615.3(345.3-906.1)$ & $1065(889.2-1215)$ & $677.5(535.6-711.1)$ & $1034(885.5-1149)$ & $900.3(382.8-1117)$ \\
\hline \multirow[t]{3}{*}{ CD8+ } & AUC (U) & $2285(2220-2444)$ & $2164(2105-2423)$ & $2258(2224-2318)$ & 2199 (2121-2667) & $2404(2265-2471)$ & $2322(1964-2566)$ \\
\hline & $\operatorname{Max}(\mathrm{rpv})$ & $1.181(1.141-1.317)$ & $1.213(1.09-1.365)$ & $1.156(1.141-1.198)$ & $1.174(1.137-1.355)$ & $1.213(1.067-1.521)$ & $1.257(1.159-1.284)$ \\
\hline & $\mathrm{t}_{\max }(\mathrm{s})$ & $648.1(519.4-1014)$ & $531.7(436.3-682.5)$ & $988.1(671.6-1076)$ & $659.5(455.8-856.5)$ & $606.6(541.9-927.9)$ & $590.2(388.9-1001)$ \\
\hline
\end{tabular}

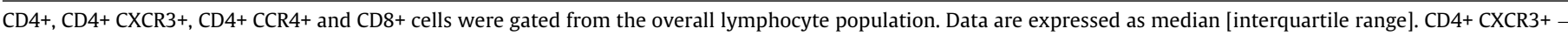

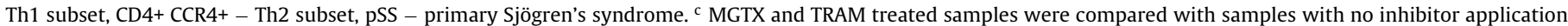
within lymphocytes isolated from pSS patients, $\mathrm{p}<0.05$.

a Lymphocytes isolated from pSS patients were compared with lymphocytes isolated from healthy individuals within samples with no inhibitor application, $\mathrm{p}<0.05$.

b MGTX and TRAM treated samples were compared with samples with no inhibitor application within lymphocytes isolated from healthy individuals, $\mathrm{p}<0.05$.

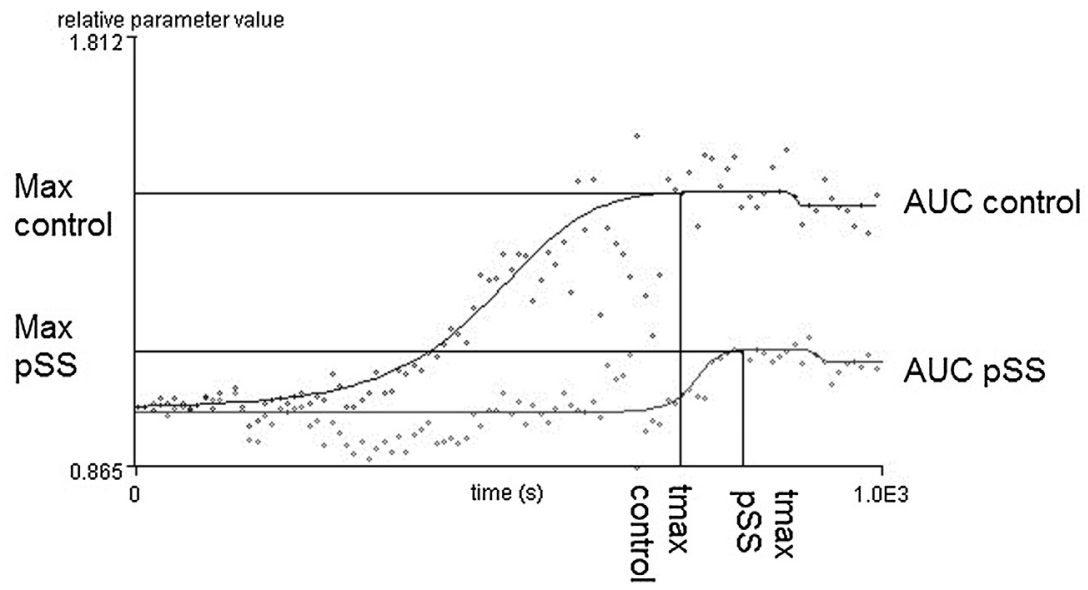

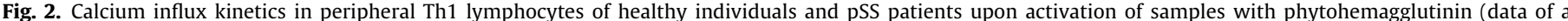

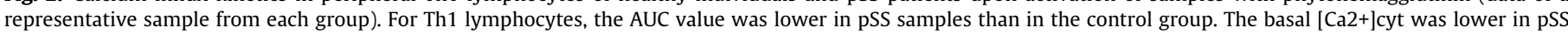

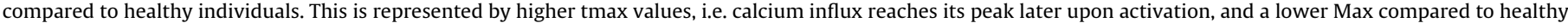
controls.

Abbreviations: pSS-primary Sjögren syndrome, AUC-area under the curve, Max-maximum value, tmax-time to reach maximum

In the pSS group, neither of the inhibitors induced alteration in calcium influx of lymphocytes (Table 3, Fig. 4).

\subsection{Kv1.3 channel expression in pSS in the investigated lymphocyte subsets}

We observed a significantly higher Kv1.3 channel expression in CD4+, Th2, and CD8+ lymphocyte subsets in pSS samples than in control lymphocytes. The median fluorescence of the study groups was similar in the case of the Th1 lymphocyte subsets. There was no difference among the several control lymphocyte subsets (Fig. 3).

\section{Discussion}

\subsection{Calcium influx kinetics during lymphocyte activation}

We applied a novel flow cytometry-based approach for the detection of calcium influx. Until the recent past, single-cell techniques were used for the investigation of calcium influx during lymphocyte activation. There has been no high-throughput method

\section{Anti-Kv1.3 FITC}

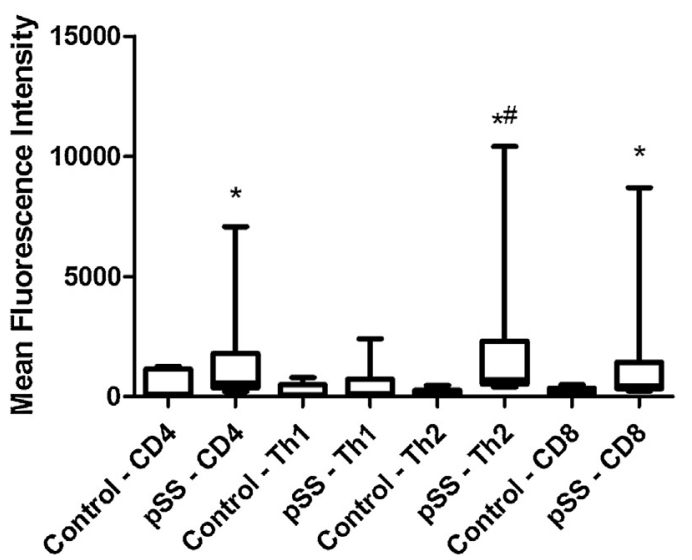

Fig. 3. Mean fluorescence intensity of the anti-Kv1.3 channel antibody in the investigated lymphocyte subsets in healthy individuals and primary Sjögren's syndrome patients. ${ }^{*} \mathrm{p}<0.05$ vs. control \# $\mathrm{p}<0.05$ vs Th1. 

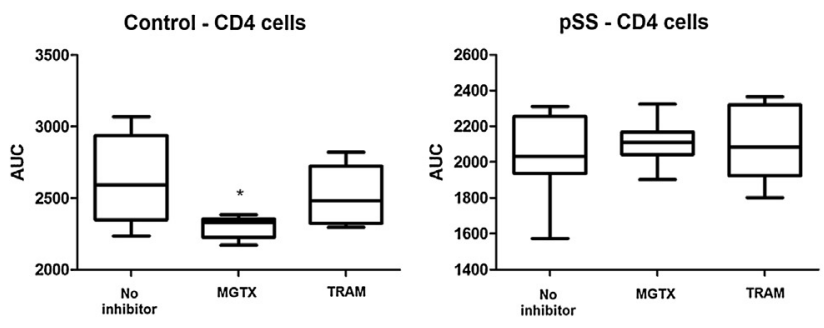

Control - Th1 cells
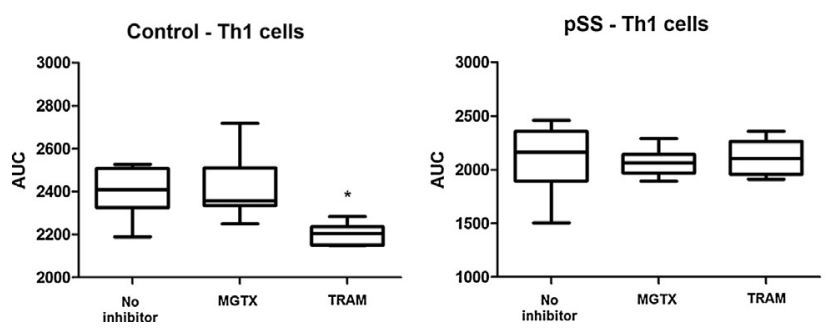
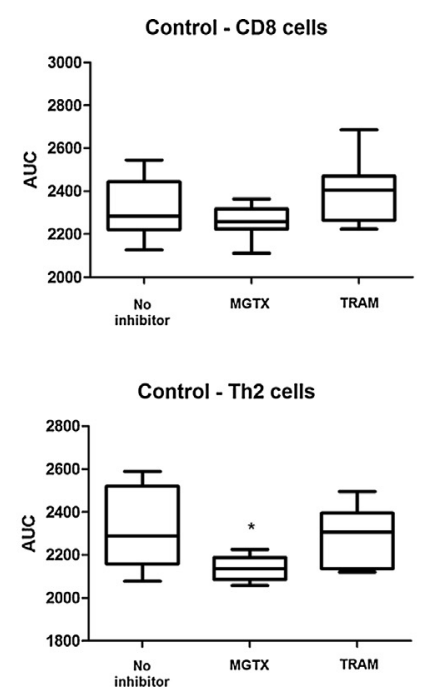

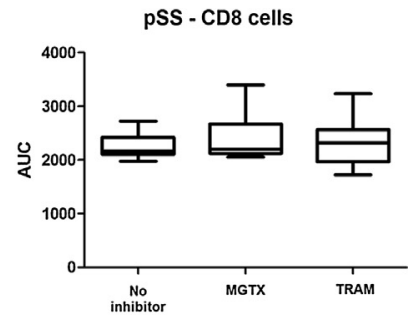

pSS - Th2 cells

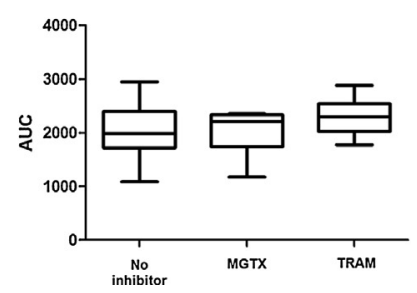

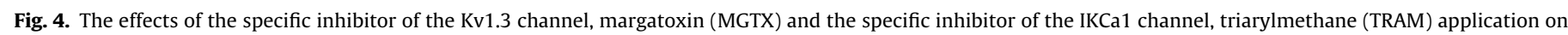

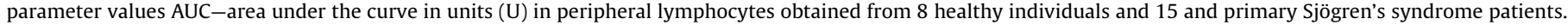
*p $<0.05$ vs. control.

available to study the kinetics of lymphocyte activation in more subsets at the same time. Single-cell techniques are restricted by not being capable of characterizing this process in complex cellular systems, thus ignoring the interaction between the different lymphocyte subsets that may modulate the course of their activation. Therefore, over the recent years we have developed a novel algorithm that allows simultaneous monitoring of calcium influx in several lymphocyte subsets. Our software (FacsKin) fits functions to median values of the data of interest and calculates relevant parameters describing each function. By selecting the best fitting function, this approach provides an opportunity for the mathematical analysis and statistical comparison of kinetic flow cytometry measurements of distinct samples (Toldi et al., 2011).

\subsection{Basal cytoplasmatic calcium levels and calcium influx kinetics in the investigated lymphocyte subsets}

Several animal models have already demonstrated that the reduced TCR signal causes increased susceptibility to autoimmune diseases. SKG mice have a genetic defect in ZAP-70, a key signal transduction molecule in T-cells leading to T-cells mediated autoimmune arthritis, and other autoimmune disorders. This defect attenuates the TCR signal therefore negative and positive selection of $\mathrm{T}$ cells is impaired in the thymus promoting self-reactive hyporesponsiveness of T-cell selection. On the other hand alloantigens induce sufficient response in ZAP-70 deficient autoreactive T-cells. Our previous observation in type one diabetes and rheumatoid arthritis are in line with animal models (e.g. collagen induced arthritis) where autoimmunity can be triggered by exuberant $T$ cell responses. However, our results in primary Sjögren syndrome are rather comparable to SKG animal models with sub-optimal T cell activation (Sakaguchi et al., 2012).

In contrast with our previous studies in autoimmune disorders, multiple sclerosis, rheumatoid arthritis, and type one diabetes, we found that lymphocytes isolated from pSS patients respond slowler to stimulation than those of healthy individuals (Toldi et al., 2013). Furthermore, the findings of our study indicate that basal [Ca2+]cyt was lower in pSS compared to healthy individuals. This is represented by higher tmax values, i.e. calcium influx reaches its peak later upon activation, and a lower AUC compared to healthy controls (Table 3, Fig. 2). This finding is especially relevant in the Th1 subset, also indicating that Th1 responses peripherally might be decreased in pSS to control the ongoing inflammation (Cornec et al., 2014).

The pathological hallmark of pSS is a chronic mononuclear cell infiltrate affecting exocrine glands mainly driven by $\mathrm{T}$ helper (Th) 1-type cytokines (Beeton et al., 2006). Recently, however, growing evidence has been suggesting that the pro-inflammatory cytokine interleukin (IL)-17 plays a pivotal role in the pathogenesis of pSS (Gong et al., 2014). Additionally, presence of different T cell subsets was evidenced in pSS, in peripheral blood and in salivary glands. At least six Th subsets exist: Th0, Th1, Th2, Th17, regulatory $\mathrm{T}$ (Treg), and follicular helper T (Tfh) cells, which are suggested to be involved in the pathogenesis of pSS (Toldi et al., 2011). The salivary glands are predominantly infiltrated by $\mathrm{CD} 4+\mathrm{T}$ helper $(\mathrm{Th})$ cells at an early stage of pSS, and in advanced stage, B cells predominate and these infiltration extends to occupy the acinar epithelium (SjögrenIgG4) (Nocturne and Mariette, 2013). Additionally, besides conventional CD4+ Th17 cells, another IL-17-producing T-cell subpopulation, lacking both CD4 and CD8 surface molecules (double negative, DN) has a key role in the pathogenesis of pSS (Alunno et al., 2014).

Furthermore, an epigenome-wide DNA methylation study identified several genes which were hypo or hypermethylated in peripheral naïve CD4+T cells from pSS patients compared to healthy controls (Altorok et al., 2014). Hypomethylated genes were mainly involved in lymphocyte activation and immune response, whereas hypermethylated genes were involved in antigen processing and presentation.

In this study, all pSS patients were enrolled after longer onset of disease (years after the presentation of the diagnosis) without any severe systemic manifestations (low ESSDAI) at time of blood sampling. Theoretically, all of these observations in pSS including the role of different Th subsets, the presence of different $\mathrm{T}$ cell subsets in peripheral blood and in salivary glands might partially explain the decreased reactivity of peripheral Th1 and Th2 lymphocytes in pSS and the absence of significant difference regarding individual cell subsets among the study groups.

Muscarinic acetylcholine receptor (M3R) is expressed in exocrine glands and plays crucial roles in exocrine secretion. Acetylcholine binds to and activates M3R on salivary gland cells, causing a rise in intracellular Ca2+ concentration via inositol 1, 4, 5-triphosphate (IP3) and IP3 receptors. Similarly to T cell activation, the increase of the cytoplasmic calcium concentration is the 
cornerstone of salivary gland cells and functionality. Several antiM3R antibodies (Abs) were described in pSS. These anti-M3R Abs cause salivary dysfunction in pSS via reduction in Ca2+ influx and down-regulation of M3R molecules on epithelial cells of salivary glands. Next to the destruction of affected tissues (mainly salivary and lacrimal glands) another feature of pSS is B cell hyperactivity, as attested by abundant production of autoantibodies (Sumida et al., 2014). Autoimmune responses are mainly polyclonal, targeting multiple epitopes within the same or interacting autoantigens. Despite extensive studies, the mechanisms operating for the exposure of certain intracellular autoantigens to the immune system remain rather elusive. It is tempting to speculate potential Abs cause Th1 and Th2 peripheral lymphocytes dysfunction via altered calcium influx kinetics in pSS.

Based on our results, the time when the peak of calcium influx was reached decreased in autoimmune patients compared to healthy individuals, indicating that these cells are in a state of sustained reactivity due to the ongoing autoimmune reaction. Contrary, not only the time when the peak of calcium influx was reached increased significantly, but the peak of calcium influx decreased significantly too, indicating that these cells are in an insensitive state which suggests the distinct pathomechanism of pSS (Table 3, Fig. 2).

\subsection{The effects of potassium channel inhibitors on lymphocyte calcium influx}

Earlier studies were limited to the investigation of potassium channels in naive and memory lymphocytes. We have extended these findings to significant effector $T$ lymphocyte subsets, and found a different pattern of sensitivity to the inhibition of lymphocyte potassium channels in Th1 cells of autoimmune patients (RA, MS, T1DM) compared to healthy individuals (Toldi et al., 2010, 2011, 2013). In the investigated autoimmune patients a greater decrease of calcium influx upon the inhibition of the Kv1.3 channel than that of the IKCa1 channel was observed in Th1 cells. This finding is of special interest, since Th1 cells are regarded as key players in the mediation of pro-inflammatory responses. However, the selectivity of the investigated inhibitors was limited in our experiments, as they did not only affect a single subset, as previously suggested. Although in earlier observations the inhibition of Kv1.3 channels specifically blocked the function of TEM cells, our investigations extending to significant effector T lymphocyte subsets demonstrated that the inhibitory effect is present not only in disease-associated CD8 and Th1 cells, but also in the anti-inflammatory Th2 subset.

However, previous data harmonizing with our results suggest that selective modulation of lymphocyte activation through specific inhibition of potassium channels may be a possible therapeutic approach for the treatment of autoimmune disease, peripheral Tlymphocytes in pSS were insensitive to the potassium channel inhibitors.

\subsection{Kv1.3 channel expression in pSS in the investigated lymphocyte subsets}

The insensitivity of peripheral T-lymphocytes in pSS to the potassium channel inhibitors results may be due to altered functionality or changes in the expression of Kv1.3 channels. We therefore analyzed cell surface expression of Kv1.3 channel by measuring median fluorescence of specific antibodies. Changes to the sensitivity of Kv1.3 channel inhibition seem to be related to its altered expression, and therefore, might explain our findings. Hence, functional alterations of the Kv1.3 channel must also play a role in differential sensitivity upon inhibition detected between pSS patients and healthy controls. Of note, due to the lack of commer- cially available antibodies against the other investigated potassium channel, we could not perform a similar measurement in case of IKCa1.

\section{Conclusion}

In conclusion, the altered expression of Kv1.3 channels and specific inhibition of potassium channels seem to be related to altered calcium influx kinetics in pSS which distinguish pSS either from healthy controls or other systemic autoimmune diseases. These observations support the differential pathomechanism of pSS. Therefore, further studies, including the analysis of other lymphocytes subsets and functional consequences of specific inhibition of potassium channels such as cytokine production would add valuable information.

\section{Conflict of interest}

All authors declare no conflict of interest related to this manuscript.

\section{Contributorship}

Study conception and design: NL, GT, ABalog; Data collection and analysis: NL, NM, CO, ABajnok; review and approve the final manuscript: GT, ABalog Analysis and interpretation of data: NL, GT, ABalog; NL, GT, ABalog wrote the manuscript.

\section{Acknowledgements}

The study was supported by the research grant of Hungarian Association of Rheumatologists (2014-2015). Attila Balog and Gergely Toldi were supported by the János Bolyai Scholarship. Gergely Toldi is an International Society for the Advanecement of Cytometry (ISAC) Scholar. The funders had no role in study design, data collection and analysis, decision to publish, or preparation of the manuscript.

\section{References}

Altorok, N., Coit, P., Hughes, T., Koelsch, K.A., Stone, D.U., Rasmussen, A., Radfar, L., Scofield, R.H., Sivils, K.L., Farris, A.D., Sawalha, A.H., 2014. Genome-wide DNA methylation patterns in naive CD4 T cells from patients with primary Sjögren's syndrome. Arthritis Rheumatol. 66, 731-739, http://dx.doi.org/10.1002/art. 38264.

Alunno, A., Carubbi, F., Bistoni, O., Caterbi, S., Bartoloni, E., Bigerna, B., Pacini, R., Beghelli, D., Cipriani, P., Giacomelli, R., Gerli, R., 2014. CD4-CD8- T-cells in primary Sjögren's syndrome: association with the extent of glandular involvement. J. Autoimmun. 51, 38-43, http://dx.doi.org/10.1016/j.jaut.2014. 01.030.

Beeton, C., Wulff, H., Standifer Azam, N.E.P., Mullen, K.M., Pennington, M.W., Kolski-Andreaco, A., Wei, E., Grino, A., Counts, D.R., Wang, P.H., LeeHealey, C.J., Andrews, S.B., Sankaranarayanan, A., Homerick, D., Roeck, W.W., Tehranzadeh, J., Stanhope, K.L., Zimin, P., Havel, P.J., Griffey, S., Knaus, H.G., Nepom, G.T., Gutman, G.A., Calabresi, P.A., Chandy, K.G., 2006. Kv1. 3 channels are a therapeutic target for T cell-mediated autoimmune diseases. Proc. Natl. Acad. Sci. U. S. A. 103, 17414-17419, http://dx.doi.org/10.1073/pnas.0605136103.

Cornec, D., Jamin, C., Pers, J.O., 2014. Sjögren's syndrome: where do we stand, and where shall we go? J. Autoimmun. 51, 109-114, http://dx.doi.org/10.1016/j. jaut.2014.02.006.

Feske, S., 2013. Ca2 + influx in T cells: how many Ca2+ channels? Front. Immunol. 4, 99, http://dx.doi.org/10.3389/fimmu.2013.00099.

Gong, Y.Z., Nititham, J., Taylor, K., Miceli-Richard, C., Sordet, C., Wachsmann, D., Bahram, S., Georgel, P., Criswell, L.A., Sibilia, J., Mariette, X., Alsaleh, G., Gottenberg, J.E., 2014. Differentiation of follicular helper T cells by salivary gland epithelial cells in primary Sjögren's syndrome. J. Autoimmun. 51, 57-66, http://dx.doi.org/10.1016/j.jaut.2013.11.003.

Nocturne, G., Mariette, X., 2013. Advances in understanding the pathogenesis of primary Sjögren's syndrome. Nat. Rev. Rheumatol. 9, 544e56.

Orbán, C., Biró, E., Grozdics, E., Bajnok, A., Toldi, G., 2013. Front. Immunol. 4, 234, http://dx.doi.org/10.3389/fimmu.2013.00234. 
G Model

IMBIO-51494; No. of Pages 7

N. Legány et al. / Immunobiology $x x x$ (2016) $x x x-x x x$

7

Rangaraju, S., Chi, V., Pennington, M.W., Chandy, K.G., 2009. Kv1.3 potassium channels as a therapeutic target in multiple sclerosis. Expert Opin. Ther. Targets 13, 909-924, http://dx.doi.org/10.1517/14728220903018957.

Sakaguchi, S., Benham, H., Cope, A.P., Thomas, R., 2012. T-cell receptor signaling and the pathogenesis of autoimmune arthritis: insights from mouse and man. Immunol. Cell Biol. 90, 277-287, http://dx.doi.org/10.1038/icb.2012.4.

Seror, R., Bowman, S.J., Brito-Zeron, P., Theander, E., Bootsma, H., Tzioufas, A., Gottenberg, J.E., Ramos-Casals, M., Dörner, T., Ravaud, P., Vitali, C., Mariette, X., Asmussen, K., Jacobsen, S., Bartoloni, E., Gerli, R., Bijlsma, J.W., Kruize, A.A., Bombardieri, S., Bookman, A., Kallenberg, C., Meiners, P., Brun, J.G., Jonsson, R., Caporali, R., Carsons, S., De Vita, S., Del Papa, N., Devauchelle, V., Saraux, A., Fauchais, A.L., Sibilia, J., Hachulla, E., Illei, G., Isenberg, D., Jones, A., Manoussakis, M., Mandl, T., Jacobsson, L., Demoulins, F., Montecucco, C., Ng W.F., Nishiyama, S., Omdal, R., Parke, A., Praprotnik, S., Tomsic, M., Price, E., Scofield, H., Sivils, L.K., Smolen, J., Laqué, R.S., Steinfeld, S., Sutcliffe, N., Sumida, T., Valesini, G., Valim, V., Vivino, F.B., Vollenweider, C., 2015. EULAR Sjégrenös syndrome disease activity index (ESSDAI): a user guide. RMD Open 1, e000022, http://dx.doi.org/10.1136/rmdopen-2014-000022.

Shiboski, S.C., Shiboski, C.H., Criswell, L., Baer, A., Challacombe, S., Lanfranchi, H., Schiødt, M., Umehara, H., Vivino, F., Zhao, Y., Dong, Y., Greenspan, D., Heidenreich, A.M., Helin, P., Kirkham, B., Kitagawa, K., Larkin, G., Li, M., Lietman, T., Lindegaard, J., McNamara, N., Sack, K., Shirlaw, P., Sugai, S., Vollenweider, C., Whitcher, J., Wu, A., Zhang, S., Zhang, W., Greenspan, J., Daniels, T., 2012. Sjögren's International Collaborative Clinical Alliance (SICCA) Research Groups: American College of Rheumatology classification criteria for Sjögren's syndrome a data-diven expert consensus approach in the Sjögren's International Collaborative Clinical Alliance cohort. Arthritis Care Res. 64, $475-487$.

Sumida, T., Tsuboi, H., Iizuka, M., Hirota, T., Asashima, H., Matsumoto, I., 2014. The role of M3 muscarinic acetylcholine receptor reactive T cells in Sjögren's syndrome: a critical review. J. Autoimmun. 51, 44-50, http://dx.doi.org/10. 1016/j.jaut.2013.12.012.
Toldi, G., 2013. The regulation of calcium homeostasis in T lymphocytes. Front. Immunol. 4, 432, http://dx.doi.org/10.3389/fimmu.2013.00432.

Toldi, G., Vasarhelyi, B., Kaposi, A., Mészáros, G., Pánczél, P., Hosszufalusi, N., Tulassay, T., Treszl, A., 2010. Lymphocyte activation in type 1 diabetes mellitus: the increased significance of Kv1.3 potassium channels. Immunol. Lett. 133, 35-41, http://dx.doi.org/10.1016/j.imlet.2010.06.009.

Toldi, G., Folyovich, A., Simon, Z., Zsiga, K., Kaposi, A., Mészáros, G., Tulassay, T., Vásárhelyi, B., 2011. Lymphocyte calcium influx kinetics in multiple sclerosis treated without or with interferon beta. J. Neuroimmunol. 237, 80-86, http:// dx.doi.org/10.1016/j.jneuroim.2011.06.008.

Toldi, G., Bajnok, A., Dobi, D., Kaposi, A., Kovács, L., Vásárhelyi, B., Balog, A., 2013. The effects of Kv1.3 and IKCa1 potassium channel inhibition on calcium influx of human peripheral T lymphocytes in rheumatoid arthritis. Immunobiology 218, 311-316, http://dx.doi.org/10.1016/j.imbio.2012.05.013.

Toldi, G., Munoz, L., Herrmann, M., Schett, G., Balog, A., 2015. The effects of Kv1.3 and IKCa1 channel inhibition on cytokine production and calcium influx of T lymphocytes in rheumatoid arthritis and ankylosing spondylitis. Immunol. Res. (August 18), Epub ahead of print.

Tzioufas, A.G., Kapsogeorgou, E.K., Moutsopoulos, H.M., 2012. Pathogenesis of Sjögren's syndrome: what we know and what we should learn. J. Autoimmun. $39,4 \mathrm{e} 8$.

Vitali, C., Bombardieri, S., Jonsson, R., Moutsopoulos, H.M., Alexander, E.L., Carsons, S.E., Daniels, T.E., Fox, P.C., Fox, R.I., Kassan, S.S., Pillemer, S.R., Talal, N., Weisman, M.H., 2002. European study group on classification criteria for sjögren's syndrome: classification criteria for Sjögren's syndrome a revised version of the european criteria proposed by the American-European consensus group (review). Ann. Rheum. Dis. 61, 554-558.

Wulff, H., Calabresi, P.A., Allie, R., Yun, S., Pennington, M., Beeton, C., Chandy, K.G., 2003. The voltage-gated $\mathrm{Kv} 1.3 \mathrm{~K}(+)$ channel in effector memory $\mathrm{T}$ cells as new target for MS. J. Clin. Invest. 111, 1703-1713, http://dx.doi.org/10.1172/ JCI16921.

Please cite this article in press as: Legány, N., et al., Calcium influx kinetics, and the features of potassium channels of peripheral lymphocytes in primary Sjögren's syndrome. Immunobiology (2016), http://dx.doi.org/10.1016/j.imbio.2016.06.004 